\title{
L'innovation en éducation, un enjeu majeur pour les pays francophones
}

Innovation in education system, a major challenge for French-speaking countries

La innovación en la educación, un desafíon importante para los paíes francófonos

\section{Didier Oillo}

\section{(2) OpenEdition}

\section{Journals}

Édition électronique

URL : http://journals.openedition.org/ctd/366

DOI : $10.4000 /$ ctd. 366

ISSN : 2491-1437

Éditeur

Chaire Unesco Pratiques émergentes en technologies et communication pour le développement

Référence électronique

Didier Oillo, "L'innovation en éducation, un enjeu majeur pour les pays francophones »,

Communication, technologies et développement [En ligne], 5 | 2018, mis en ligne le 02 janvier 2018,

consulté le 21 avril 2019. URL : http://journals.openedition.org/ctd/366 ; DOI : 10.4000/ctd.366

Ce document a été généré automatiquement le 21 avril 2019

Communication, technologies et développement 


\title{
L'innovation en éducation, un enjeu majeur pour les pays francophones
}

\author{
Innovation in education system, a major challenge for French-speaking \\ countries \\ La innovación en la educación, un desafíon importante para los paíes \\ francófonos
}

Didier Oillo

1 Il s'agit ici de se questionner sur le nécessaire changement éducatif dans les pays francophones les moins avancés. Face à une démographie galopante, il est banal de dire que les méthodes traditionnelles d'enseignement ne sont plus adaptées. Les technologies peuvent-elles être une alternative à un enseignement tout à la fois de qualité et de masse dans les Suds, notamment en Afrique, sujet de notre réflexion?

2 Nous aborderons notre propos par un constat démographique et nous émettrons l'hypothèse que les innovations de rupture en pédagogie peuvent apporter une solution si elles ne sont pas considérées seulement dans leur aspect expérimental.

\section{Un monde en mutation}

Lorsqu'on lit différents rapports notamment de l'Organisation internationale de la Francophonie (OIF) sur la démographie en Francophonie, on constate que les prévisions pour 2050 interpellent les éducateurs que nous sommes. Non seulement la population mondiale passera de 6,7 milliards en 2005 à 9,2 en 2050, mais sa structure d'âge sera grandement modifiée. Le nombre de personnes en âge de travailler (15-64 ans) passera de 4,2 milliards en 2005 à 5,9 milliards en $2050^{1}$. La répartition des populations sera également très différente. De $22,2 \%$ de la population mondiale en 2005 , le pourcentage de la Chine ne sera plus en 2050 que de $14,6 \%$. De $11 \%$ en 2005, l'Europe baissera à $8,2 \%$ en 2030 et à $6,5 \%$ en 2050 , ce qui ne manquera pas d'induire des besoins nouveaux en immigration à tous les niveaux. 


\section{Et l'Afrique?}

4 La part de l'Afrique dans la population mondiale en âge de travailler évoluera de 12,1\% en 2005 à $17 \%$ en 2030, pour atteindre $22,1 \%$ en 2050. Á l'examen de son potentiel démographique, il paraît évident que l'Afrique, si elle réussit son intégration économique continentale, disposera de tous les atouts pour devenir, dans les prochaines décennies, le principal relais de croissance mondial, plus encore que ne l'a été la Chine ces vingt dernières années. Contrairement à ce qu'on pourrait imaginer en analysant ses ressources naturelles, c'est bien dans ses ressources humaines que se situe le principal capital du continent africain.

\section{Un nouvel ordre mondial}

5 Alors qu'aujourd'hui l'Afrique peine à former sa jeunesse, comment imaginer qu'elle pourra assumer une telle croissance démographique ? Quels sont les scénarios d'avenir ? Pouvons-nous anticiper sur la création d'une économie basée sur la connaissance si souvent abordée dans une perspective économique, au détriment de sa dimension culturelle et citoyenne, en ignorant les implications sociales de l'innovation, et en nous privant de quantité d'expérimentations et de savoirs?

6 De plus, dans un système global multipolaire, tout circule, les idées, les étudiants, les enseignants, les chercheurs. Le théâtre des opérations est en mutation constante, il se redéfinit avant qu'on ait pu le cerner, il élargit en permanence son territoire. De 100 millions d'étudiants en 2000, il y en aura 200 millions en 2015. De plus en plus mobiles, les étudiants furent, en 2008, 3 millions environ à avoir étudié hors de leur pays d'origine. On en comptera autour de 10 millions en 2030. Que vont devenir les talents africains de plus en plus attirés par les offres alléchantes des prestigieuses universités et Grandes écoles du Nord?

$7 \quad$ Il est temps de réfléchir à ces mutations profondes qui impliquent de regarder la société autrement. Une société de la connaissance partagée est-elle envisageable? Sur quelles bases et sur quelles valeurs ? À partir de quelles expériences ? Pour quels bénéficiaires?

8 Á ces questions auxquelles nous ne prétendons pas apporter de réponses immédiates, il en reste une, fondamentale pour la Francophonie: quelle sera la langue de communication de ces populations nouvelles? On ne peut établir un parallélisme entre croissance démographique des pays membres de l'OIF et croissance des locuteurs francophones. Le risque est grand. D'ores et déjà, on peut observer de nouveaux choix linguistiques des États. À titre d'exemple, le Burundi retient quatre langues d'enseignement, le kirundi, le kiswahili, l'anglais et le français. La Francophonie qui promeut le multilinguisme ne peut certes que se féliciter de ces choix. Mais qu'en sera-t-il si le français ne fait plus partie des langues d'enseignement?

Il faut que la Francophonie apporte à ces États des éléments qui la rendent concurrentielle et qui lui donnent l'avantage. Un des points qui nous semble le plus important est l'éducation. C'est un des secteurs dans lequel la Francophonie dispose, à travers ses opérateurs et les coopérations bilatérales de ses États membres, d'une longue expérience. Cet avantage compétitif, la Francophonie doit le trouver dans l'innovation. 

pratiques ou répondre à de nouveaux enjeux. Il s'agit d'aborder une vision prospective du système éducatif et d'en favoriser les expérimentations.

En pédagogie, comme dans bien d'autres domaines, nous devons refuser l'idée qu'il existe une solution universelle aux problèmes de l'éducation. Il faut préserver le principe de diversité tant au niveau des approches que des contenus. Sans quoi, nous risquons au nom des économies d'échelle et de l'uniformisation des exigences, de voir les dispositifs pédagogiques se standardiser de plus en plus, et, à terme, se scléroser².

Trois grands champs déterminent l'innovation pédagogique :

- les caractéristiques techniques des outils,

- celles plus fonctionnelles de l'institution ou de l'organisation et,

- celles du comportement des individus ayant une activité en situation ${ }^{3}$.

Dans cette dernière catégorie, les individus sont définis en tant qu'acteurs. Afin d'aborder la «fabrique de l'innovation ", nous écarterons momentanément la vitrine technologique. C'est la pédagogie qu'il faut d'abord réinventer en s'appuyant sur les possibilités offertes par les technologies, car celles-ci ne sont que des moyens au service des finalités de l'enseignement.

Nous devons prendre en compte à la fois les directives officielles et le fonctionnement de l'institution, la voie officielle, d'une part, et la voie officieuse, faite d'innovations individuelles, que nous qualifions d'innovations de rupture, d'autre part. Ces deux voies n'obéissent pas aux mêmes logiques mais concourent à l'innovation.

15 Nous séparons donc, de façon arbitraire, l'innovation institutionnelle de celle, plus intuitive, des pionniers. Par exemple, les universités européennes, élargies à de nombreuses universités de l'espace francophone connaissent le processus de Bologne qui a abouti à la création du LMD (Licence, Master, Doctorat). Plus rares sont ceux qui maitrisent le concept de formation ouverte et à distance voire de formation hybride et plus récemment de formations massives. Dans chacun de ces exemples on perçoit des éléments de transformation de l'éducation, prise au sens large, au début du XXI ${ }^{\mathrm{e}}$ siècle.

Deux analyses sont nécessaires à la compréhension de ces deux importants mouvements, l'une macroanalytique, s'appuyant sur des textes (exemple: la réforme LMD) et l'autre microanalytique menée à partir de l'observation de projets (l'introduction des Techniques d'Information et de Communication pour l'Enseignement - TICE - dans l'université et plus largement d'ans l'éducation).

\section{Les pionniers}

Rappelons que c'est en 1840 qu'Isaac Pittman créait en Angleterre les premiers cours d'enseignement par correspondance, suivi, en 1877, de Rose Hattemer en France.

Dans les années 1970, les francophones marquent le pas par la création du Conseil international de l'enseignement à distance, du Consortium international francophone de formation à distance (CIFFAD), de l'Association des écoles européennes d'enseignement par correspondance et de l'Association des universités ouvertes d'Asie. C'est en 1972 que naît l'Open university devenue l'archétype de la formation ouverte et à distance ${ }^{4}$. 


\section{La Francophonie faisait partie des pionniers}

\section{Des expérimentations en Francophonie}

19 Repris et renforcé donc par l'actuelle Organisation internationale de la francophonie (OIF), le CIFFAD, incorporé à sa programmation en 1990, est un réseau d'établissements ayant pour objectifs la consolidation des dispositifs de formation à distance, la création et l'adaptation de programmes et la dynamisation des capacités nationales et régionales en formation à distance. Ce consortium, né avant la généralisation de l'Internet, a établi dès sa naissance des liens avec de grandes organisations internationales telles que l'UNESCO, l'Islamic educational, scientific and cultural organisation (ISESCO), le Commonwealth of Learning (COL), l'Association africaine francophone de formation à distance (ASAFFAD), renforçant ainsi sa capacité de réflexion. Forts du relatif échec de la télévision éducative de Bouaké, qui fut pensée depuis la France pour l'Afrique et qui nécessitait des moyens techniques considérables non disponibles dans le pays, les membres du consortium ont adopté l'idée que toute action qui impliquait un changement de type technologique devait obligatoirement être accompagnée d'un ensemble de mesures de formations permettant une véritable prise en charge de ladite technologie par les partenaires locaux. Il est très vite apparu qu'il était essentiel de bien planifier les opérations afin d'adapter l'implantation de tout programme à caractère technologique au rythme d'installation des infrastructures locales et ainsi d'éviter la création d'un fossé technologique.

Le CIFFAD a cherché à préserver les valeurs pédagogiques les plus fondamentales des pays de la francophonie en connaissant et respectant les paradigmes pédagogiques en vigueur dans chacun d'eux. Partant du principe que les sociétés actuelles, tant au Nord qu'au Sud, connaissent d'importantes modifications de leurs systèmes éducatifs sans pour autant que cette mutation se fasse au même rythme et selon la même progression partout. Dans les pays francophones en voie de développement, le modèle «traditionnel », qui accorde une place prépondérante à l'acte d'enseignement et à l'enseignant, est encore fortement présent par opposition aux nouveaux paradigmes éducatifs (constructivisme, phénoménologisme voire connectivisme) qui placent davantage l'apprentissage et l'apprenant au centre de l'acte pédagogique dans plusieurs pays francophones développés. Par ailleurs, s'il est une valeur pédagogique importante qui doit nécessairement être respectée dans la coopération en formation à distance c'est bien la nécessité de voir la technologie au service des programmes. On ne le répétera jamais assez, nous avons trop souvent vu des artifices technologiques se présenter comme des panacées à la formation à distance alors qu'ils ne prenaient pas en compte la portée pédagogique de leurs moyens. À titre d'exemple on peut citer le projet de radio numérique lancé par le groupe Wolrdspace.

21 C'est au sein du CIFFAD qu'ont été pensés de nombreux programmes de formation à distance dont ceux de l'AUF ou encore du canal éducatif francophone et du Réseau africain de formation à distance (RESAFAD), conçu et piloté par le très regretté Jean Valérien. 


\section{La Francophonie vecteur d'innovation. La mise à distance des savoirs, une approche timide mais réelle} cours duquel se réalise la finalité de tout dispositif de formation : apprendre. Apprendre est donc au cœur de toutes les pratiques éducatives. Par ailleurs, la conduite d'une formation à distance se distingue de la conduite d'une formation traditionnelle par au moins deux points : la désynchronisation partielle des relations entre l'apprenant et le(s) formateur(s) et le polymorphisme de la conduite. Lors d'une formation en présentiel, le formateur dispose de divers indicateurs qui lui permettent, non seulement de mesurer l'impact de son enseignement sur l'apprenant, mais surtout de remédier instantanément à toutes pertes de l'attention, aux incompréhensions ou aux réactions face à son discours. Á distance, ces indicateurs spontanés n'existent plus et il est indispensable d'en imaginer d'autres et de trouver un compromis sur la durée, naturellement plus longue, entre l'émission d'un message d'alerte ou d'une demande d'aide et sa résolution.

Le polymorphisme de la conduite passe par des situations plus diversifiées et qui sont animées par un plus grand nombre d'acteurs, de professionnels aux rôles et fonctions diversifiés qu'il convient de préciser. Nous référant à la métaphore musicale, nous pourrions dire que le nouveau rôle « d'homme-orchestre » du formateur laisse place à la polyphonie dans laquelle les autres apprenants prennent aussi une place singulière et nouvelle dans la construction des apprentissages individuels. La formation devient une combinatoire de ces différentes situations, de manière libre ou imposée. Elle peut toutefois, sans être individualisée, proposer des parcours différenciés prédéfinis. Dans ce cas, ceux-ci doivent être explicitement décrits, et tous les éléments permettant de faire le choix le plus approprié doivent être fournis (par exemple : tests de positionnement et de capacité, entretien...).

24

Onconstatecependantquel'institutionnelsepréoccupeprioritairementdescontenusàtransmettre et peu des modalités de leur transmission, encore moins des modes d'appropriation. Cependant, il nous semble fondamental qu'une rupture avec l'apprentissage scolaire soit clairement définie. Les pionniers de l'innovation formulent des propositions que Brigitte Albero, Monique Linard et Jean-Yves Robin proposent de classer en cinq points ${ }^{5}$. En résumé : cesser de dispenser des cours magistraux à très faible rentabilité ; mettre fin à l'assistanat par groupe de niveaux ; responsabiliser les élèves et les étudiants, les rendre plus autonomes tout en les aidant; fournir un espace de travail ouvert, riche en ressources et en outils performants et riche d'une forte diversité humaine; faciliter non seulement l'appropriation des contenus mais aussi l'acquisition des modes fondamentaux de raisonnement et d'argumentation.

Dans la réalisation de ces cinq points, les technologies deviennent un support indispensable et s'imposent aux étudiants comme à leurs maîtres. De ce fait, l'intégration des TIC dans l'institution est un moyen de repenser globalement les services. La distribution des espaces et l'organisation des activités étant grandement bouleversées, la formation à distance peut prendre une place complémentaire aux enseignements présentiels. On parle alors de formations hybrides.

Concernant le supérieur, la "mise à distance » des enseignements pourrait devenir progressivement une solution, non seulement pour satisfaire aux besoins des étudiants 
éloignés ou pallier l'encombrement des équipements souvent insuffisants dans beaucoup de pays, mais également comme moteur de la transformation du paradigme traditionnel. Cependant, même s'il est convenu que les formations s'hybrident de plus en plus, elles n'impactent pratiquement pas l'offre traditionnelle et ses modes de transmission. L'université du Nord utilise encore les TICE comme vitrine de la modernité ${ }^{\text {quand, au }}$ Sud, il ne s'agit que d'expérimentations souvent difficiles à mettre en œuvre.

Dans le domaine de l'enseignement de base, la Francophonie a valorisé son expérience et les compétences de ses opérateurs pour créer l'Initiative francophone pour la formation à distance des maîtres (IFADEM).

Cette initiative est considérée comme unique en francophonie car elle s'appuie sur un dispositif cohérent, alliant les performances technologiques des campus numériques, la formation des formateurs, la mise à disposition de ressources éducatives libres de droit, les travaux de recherche du réseau qu'elle soutient depuis le Sommet mondial sur la société de l'information, son investissement dans la normalisation des systèmes éducatifs et un réseau d'établissements solidaires. C'est la cohérence de ce dispositif, salué lors des Sommets de Ouagadougou et de Bucarest, qui ont amené les décideurs de la Francophonie à lui confier, en partenariat avec l'OIF, un projet de formation des maîtres, l'IFADEM. Lancée sous l'impulsion du Secrétaire général de la Francophonie, le Président Abdou Diouf, celle-ci est expérimentée d'abord dans quatre pays: Bénin, Burundi, Haïti et Madagascar, et s'étend peu à peu dans de nombreux autres. Elle a pour objectifs, à court terme, d'agir dans douze pays. Elle enregistre actuellement plus d'une vingtaine de demandes. L'IFADEM se donne pour objectif principal l'amélioration des compétences des enseignants $\mathrm{du}$ primaire dans le domaine de l'enseignement $\mathrm{du}$ français par l'intermédiaire d'un dispositif de formation continue en partie à distance utilisant les TICE. La mise en œuvre d'une formation continue d'instituteurs en exercice tient compte des spécificités éducatives, socioculturelles et sociolinguistiques respectives des pays concernés. Le français y joue un rôle important dans la formation scolaire des personnes, pour qui, individuellement, très souvent il a un statut de langue étrangère (FLE), quand, sur un plan officiel, il a un statut de langue seconde (FLS).

Tous les acteurs s'accordent actuellement sur la nécessité de prendre en compte la complexité de ces contextes bi ou plurilingues dans l'enseignement pour assurer la réussite des élèves. Le dispositif prévoit environ 200 heures de formation étalées sur 9 mois. Ce parcours est rythmé par trois regroupements de 2 ou 3 jours : en début, au milieu et en fin de formation. Le dernier regroupement peut être consacré à une évaluation sommative. La formation se déroule essentiellement à distance, mais elle est accompagnée par un tutorat de proximité (tutorat réalisé par des inspecteurs, des directeurs d'école, des conseillers pédagogiques selon les pays) qui permet d'accompagner le travail des enseignants et d'organiser des rencontres par petits groupes d'instituteurs. Le dispositif intègre l'installation d'Espaces IFADEM, équipés d'ordinateurs connectés à l'Internet et spécialement installés dans des établissements de formation des maîtres. Dans ces Espaces sont organisés, pendant les regroupements, des ateliers d'initiation à l'informatique et à l'Internet. Les équipements peuvent également être utilisés pour présenter des ressources audiovisuelles et servent à la formation des élèves instituteurs hors du contexte de l'IFADEM. Chaque enseignant concerné par l'IFADEM reçoit en dotation pédagogique un dictionnaire, une grammaire (à choisir par l'expert technique et le groupe des rédacteurs de contenus), un MP3 avec des ressources audio préalablement enregistrées (selon les besoins) et des modules de formation édités sous 
forme de livrets également déclinés en ligne sur une plateforme d'enseignement à distance. Chaque module du programme de formation à distance est le fruit d'une étroite collaboration entre une équipe de linguistes-didacticiens (experts IFADEM issus des universités francophones) et une équipe de rédacteurs locaux, préalablement identifiés pour leur expérience et leurs compétences en didactique du français.

L'IFADEM repose sur les principes forts d'intégration, de conscientisation et de valorisation des compétences des instituteurs. On part d'une interrogation sur les pratiques de classe des enseignants, on apporte des savoirs nécessaires qu'on intègre, dans des activités variées, à des savoir-faire spécifiques et enfin on propose une remédiation à expérimenter dans la classe. Dans le même ordre d'idée, une démarche d'évaluation formative est intégrée à la conception des modules. Dès le départ, à l'aide d'un diagnostic et, progressivement, tout au long de la lecture des modules, les enseignants sont amenés à s'auto-évaluer, à s'approprier leur formation. Á terme, il s'agit de rendre leurs pratiques de classe plus cohérentes.

Il reste cependant à prendre en compte la massification des apprentissages et ce en quoi les TICE peuvent répondre à ce besoin.

\section{Conclusion et propositions}

En conclusion, la Francophonie institutionnelle, à travers ses opérateurs, s'est engagée résolument dans l'innovation qu'elle considère comme un levier lui permettant d'acquérir un avantage compétitif en répondant aux nouveaux besoins en éducation des États francophones. Le choix d'ancrer cette innovation permanente dans les technologies de l'information et de la communication procède d'une double logique, capitaliser sur un existant de plus de deux décennies et intensifier la réflexion et les actions sur l'inclusion des États francophones du Sud dans la société de la connaissance.

La Francophonie doit mener une fois de plus une politique volontariste dans le domaine de l'éducation. On pourra retenir quelques pistes de réflexion et de travail. Celles-ci s'appuient sur les nombreux rapports et expérimentations de ces cinq dernières années :

- rédiger un livre blanc sur l'éducation dans le monde francophone émergeant à l'issue d'une conférence internationale,

- s'appuyer sur les expérimentations comme celles de l'IFADEM ou du projet École et langue nationale en Afrique (ÉLAN) entre autres, pour créer un institut de formation continue et de certification internationale des enseignants et des personnels de l'éducation,

- y associer la Conférence des ministres de l'Éducation des États et gouvernements de la Francophonie (CONFEMEN) et l'ensemble des opérateurs qui mènent depuis des années des actions très significatives,

- créer, à l'instar du Fonds francophone des inforoutes (FFI), un fonds pour l'innovation pédagogique à destination des publics terminaux (instituteurs, conseillers pédagogiques, inspecteurs pédagogiques...). Il ne s'agit pas de financer des structures mais d'aider les innovateurs de terrain,

- intensifier les efforts de recherche, tel que le fait le projet d'observation des pratiques enseignantes en relation avec les apprentissages des élèves (OPERA), qui se déroule sur trois ans au Burkina Faso, en s'appuyant sur la création d'un réseau d'acteurs et sur des lieux de réflexion tels que l'institut international de la Francophonie, 
- appuyer les États pour qu'ils intègrent les technologies dans leur planification éducative. Il s'agit d'utiliser la technologie à des fins de renforcement qualitatif et quantitatif des capacités des enseignants.

\section{BIBLIOGRAPHIE}

Albéro Brigitte, Linard Monique, Robin Jean-Yves, Petite fabrique de l'innovation à l'université, L'Harmattan, collection Logiques sociales, 2008.

Albéro Brigitte, Thibault Françoise, E-learning et enseignement universitaire en France, CRUI, CPU, FVU, 2006.

Benveniste Annie, Mignot-Lefebvre Yvonne, « Côte d'Ivoire : télévision extra-scolaire pour l'éducation des adultes ruraux. Un bilan critique », Revue Tiers Monde, 1979, pp. 465-478.

Bloom Benjamin et al., Taxonomie des objectifs pédagogiques, vol. 1 : Domaine cognitif, Presses de l’Université du Québec, 1975.

Charlier Bernadette, Perraya Daniel, Technologie et innovation en pédagogie. Dispositifs innovants de formation pour l'enseignement supérieur, Bruxelles, De Boeck, 2003.

Carré Philippe, Moisan André et Poisson Daniel, L'Autoformation, Paris, PUF - Pédagogie d'aujourd'hui, 1997.

Crochet Marcel, Le processus de Bologne. Aboutissement d'un long processus, Études 2004/5, Tome 401, p. 461-472.

Depover Christian, Pour tirer le meilleur profit des technologies, c'est la pédagogie qu'il faut réinventer, Actes du colloque Initiative, AUF, 2001.

Henry France, Carol Lundgren, Apprentissage collaboratif à distance, Sainte Foy Presses de l'université du Québec, 2001.

Guillou Michel, Phan Trang, La troisième mondialisation, Paris, Belin, mai 2011.

Marot Jean-Claude et Darnige Anne, La téléformation, collection « Que sais-je ? » n 3168, Déc. 1996

Loire Pierre-Jean et Oillo Didier, Histoire d'un dispositif francophone de formation ouverte et à distance, Distance et savoir, vol. 4, 2007, p. 113-121.

Wolton Dominique, Il faut sauver la communication, Flammarion, 2005.

\section{NOTES}

1. Division de la population de l'ONU.

2. Depover, 2001 et 2008.

3. Albéro, Linard et Robin, 2008.

4. Marot, Darnige, 1996.

5. Albéro, Linard, Robin, 2008.

6. Albéro, Thibault, 2006. 


\section{RÉSUMÉS}

Le terrain de notre questionnement est celui de la francophonie institutionnelle, notamment celle des Suds, sur le nécessaire changement éducatif dans les pays les moins avancés. Face à une démographie galopante, il est banal de dire que les méthodes traditionnelles d'enseignement ne sont plus adaptées. Les technologies peuvent-elles être une alternative à un enseignement tout à la fois de qualité et de masse, notamment en Afrique, le continent dont l'essor démographique important n'est pas en relation avec son développement économique.

Nous aborderons notre propos par un constat démographique et nous émettrons l'hypothèse que les innovations de rupture en pédagogie peuvent apporter une solution si elles ne sont pas considérées seulement dans leur aspect expérimental et qu'elles tendent vers un passage à l'échelle.

We will question the institutional Francophonie, especially that of the South, on the necessary educational change in the least developed countries. Faced with very important demography growth it is commonplace to say that traditional teaching methods are no longer appropriate. Can technologies be an alternative to education of both quality and mass, especially in Africa, the continent whose major population growth is not related to its economic development? We will approach our subject with a demographic observation and we will hypothesize that breakthrough innovations in pedagogy can provide a solution if they are not only considered in their experimental aspect, and support the massification of education.

É a francofonia institucional, nomeadamente dos Suls, a área do nosso perguntamento, sobre a necessária mudança educativa nos paises menos advançados. Confrontados a um crescimento demográfico rápido, é banal afirmar a inadaptação actual dos metódos tradicionais de ensino. Podem as tecnologias novas ser uma alternativa ao um ensino de qualidade e no mesmo tempo de massa, particularmente em África, continente onde o crescimento demográfico não concorda com o seu desenvolvimento económico?

Abordaremos nossas palavras com um estado de lugar demográfico, e emitiremos a hipótese que as inovações de ruptura em pedagogia podem contribuir a uma solução si são consideradas não só os seus aspectos experimentais, mas também que elas permitem ser generalizadas ao mais grande número.

\section{INDEX}

Mots-clés : francophonie, numérique, pédagogie, innovation

Keywords : francophonie, digital, pedagogy, innovation

\section{AUTEUR}

\section{DIDIER OILLO}

Ancien directeur de programme AUF 